\title{
APPLICATION OF NMR MICROSCOPY TO THE MORPHOLOGICAL STUDY OF THE SILKWORM, Bombyx mori, DURING ITS METAMORPHOSIS
}

\author{
M. MAPElli*, F. GreCo*, M. Gussoni, $\dagger$ R. Consonni* AND L. ZetTA* \\ *Istituto di Chimica delle Macromolecole, CNR, Milano, Italy †Università di Milano, \\ Dipartimento di Scienze e Tecnologie Biomediche, Milano, Italy
}

\begin{abstract}
Zero (ZQ) and double (DQ) quantum 2D chemical shift selective and spin-echo 3D NMR imaging at microscopy resolution, has been applied to the morphological study of silkworm, Bombyx mori, during its metamorphosis. Attention has been focused on the evolution of the internal structure of the insect during its postembryonal life occurring through the larval, pupal and adult development. A major objective of this work was the characterization of the silk glands, responsible for synthesis and secretion of fibroin and sericin, through the changes of distribution and mobility of water, by imaging the water protons during postembryonal life stages. Moreover, alanine deriving from silk gland proteins was imaged during the last life stage of Bombyx mori. CO 1997 Elsevier Science, Inc.
\end{abstract}

Keywords: Magnetic resonance imaging; ZQ and DQ NMRI; Microscopy; Silkworm, Bombyx mori; Fibroin.

\section{INTRODUCTION}

As documented in the literature, ${ }^{1.2}$ NMR imaging (NMRI) for biological and medical studies, and material science is a well established technique. Compared with other methods, NMRI offers the advantage that image information is obtainable concerning surface as well as structural aspects of the interior of examined objects, without sample damage. The non-destructive nature of the method makes it an attractive option in studies of organ histochemistry of small animals to identify morphological and structural details and localize the major constituents, such as water, lipids and macromolecules distributed in well defined compartments. Among the applications of NMRI to animal tissue studies, a number of investigations are reported on embryo development of insects in vivo, such as locust embryo and tobacco horn worm. ${ }^{3,4,5}$ A remarkable feature of these works is the separate imaging of water and lipid distribution in the same object, delineating anatomical characters and demonstrating how the dynamics of embryogenesis can be monitored in vivo.
Recently, comparative ${ }^{31} \mathrm{P}$ and ${ }^{1} \mathrm{H}$ NMRI experiments were carried out to provide $\mathrm{pH}$ maps from the midgut of Lepidopteran larvae. ${ }^{6}$

In this paper, in order to study the silkworm, Bombyx mori, during its metamorphosis, 3D spin-echo imaging and $\mathrm{ZQ}$ and DQ filtered (1D spectroscopy and $2 \mathrm{D}$ imaging) techniques were applied. Attention has been focused on the evolution of the internal structure of the insect during four phases of its postembryonal life, that occurs through the larval, pupal and adult stages. Investigation has been addressed to the silk glands, whose posterior and middle regions synthetize and secrete fibroin and sericin, the two silk proteins responsible for the unique tensile strength and elasticity of silk fibre.

With the termination of cocoon spinning, silk glands are known to be subject to catabolic and then degenerative changes, finally disappearing as organs about 48 $h$ after larval-pupal ecdysis. ${ }^{7}$ The occurrence of an enzyme, the fibroinase, which hydrolyzes the fibroin molecules remaining in lumen contents of silk glands, has been demonstrated by Sumida et al. ${ }^{8.9}$ According to
RECEIVED 3/6/96; ACCEPTED 12/13/96.

Address correspondence to Dr. Lucia Zetta, Lab. NMR, ICM, Consiglio Nazionale delle Ricerche, via A. M. Am- père, 56, 20131 Milano, Italy. E-mail: lucy@labnmr.icmnmr.mi.cnr.it 
these authors, the physiological function of fibroinase is the hydrolysis of fibroin to produce amino acids then reabsorbed by the gland cells. Thus, these metabolites are possibly liberated into the hemolymph to be utilized in the formation of tissues or organs of the next larval or adult stages. The high activity of fibroinase was demonstrated in vitro in extracted enzyme preparations from silk glands in the "fourth molt stage and at day 1 , pharate adult" in silkworm. ${ }^{\text {" }}$

In the present study NMRI was employed not only to observe in vivo morphological modifications of silk glands, but also to evaluate changes in the water mobility, that occur during postembryonal life stages, correlated to protein mobility and fibroinase activity. Moreover ZQ and DQ filtered $1 D$ and NMRI sequences have been implemented to select the signals of the methyl moiety of alanine, the most abundant residue of fibroin.

\section{METHODS}

All NMRI experiments on Bombyx mori have been carried out on a Bruker AM 200 NMR spectrometer, using a 4.7 Tesla superconducting magnet with $9 \mathrm{~cm}$ diameter vertical bore, equipped with a 25 or $15 \mathrm{~mm}$ insert accepting 20 and $10 \mathrm{~mm}$ NMR tubes respec- tively. Orthogonal field gradient coils, built into the micro-imaging probehead, were capable of achieving gradients up to $50 \mathrm{G} / \mathrm{cm}$, with trigger pulses for switching gradients of $5 \mu$ s. The unlocked field frequency was set on the $\mathrm{H}_{2} \mathrm{O}$ resonance.

The pulse and gradient sequence used for 3D images, relative to $128 \times 128 \times 128$ matrices, was the one standardly supplied by Bruker. For all 3D spinecho experiments the acquisition parameters were: spectral width of $33 \mathrm{kHz}$; echo delay time (TE) of $6.95 \mathrm{~ms}$ and recycle time (TR) of $520 \mathrm{~ms}$. The receiver gain (RG) was always set to 16 . Short TE and TR were used in order to acquire a 3D image of an immobile silkworm, during one of its sleeping periods, lasting about one day each. Acquisition parameters were then standardized to collect 3D images during the other metamorphosis stages. The different voxel resolutions, related to the sample dimensions, and other parameters are reported in figure captions. To improve the signalto-noise ratio, in some cases up to 10 experiments were accumulated, for a total experimental time of about one day. Such acquisition time, even if long, was anyway compatible with the slow morphological changes that would have been monitored. A pulse sequence for gradient-enhanced alanine editing ${ }^{10}$ was used to obtain ZQ and DQ filtered spectra (Scheme 1A). The method, suitable for $\mathrm{AX}_{3}$ spin systems, selects both $\mathrm{ZQ}$ and

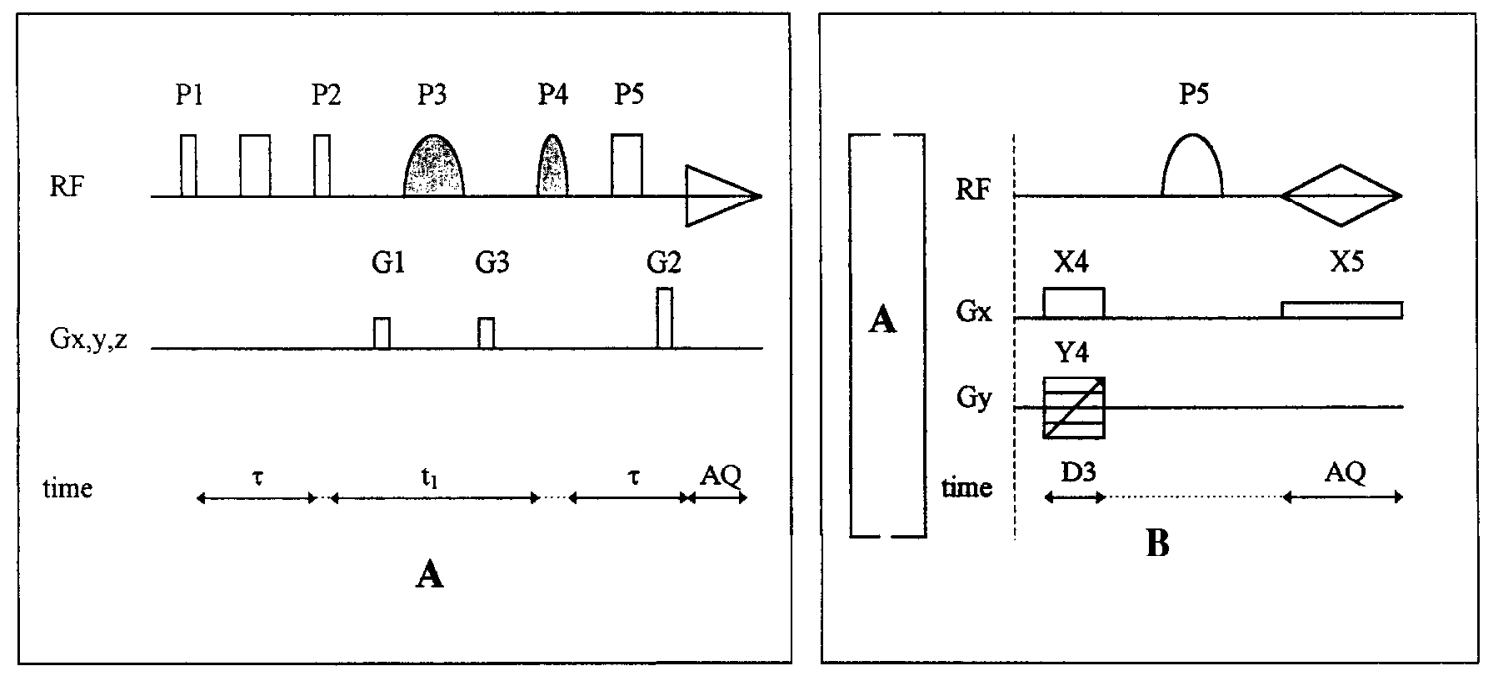

Scheme 1. Left A): $1 \mathrm{D} Z \mathrm{ZQ}$ and DQ gradient-enhanced alanine selective sequence. $\tau=1 / 2 \mathrm{~J}_{\mathrm{CH}-\mathrm{CH}_{3}}=68.4 \mathrm{~ms}$, corresponding to a $\mathrm{J}_{\mathrm{CH}-\mathrm{CH} 3}=7.32 \mathrm{~Hz} ; \mathrm{P} 3$ (dashed) and $\mathrm{P} 4$ (dashed) are binomial 11 selective pulses $(\pi$ and $\pi / 2$ respectively) on the alanine methine resonance, with an interpulse delay of $1 / 2 \Delta \delta=1.084 \mathrm{~ms}, \Delta \delta$ being the chemical shift difference between the alanine methyl and methine resonances; the simmetric gradients, $\mathrm{G} 1=\mathrm{G} 3(2.54 \mathrm{G} / \mathrm{cm})$ together with P3, cause both zero and double quantum coherences to be equally encoded. The coherences are refocused after P5 ( $\pi$ hard pulse) by G2 $=(\mathrm{G} 1+\mathrm{G} 3$ ). The single quantum water coherences possibly present in the transversal plane during the evolution period $t_{1}$ will be equally phase encoded by $\mathrm{Gl}$ and $\mathrm{G} 3$ and refocused by $\mathrm{G} 2$. A time $T=2 \tau+t_{1}=145 \mathrm{~ms}$ elapses between the excitation pulse P1 and the beginning of acquisition period $A Q$. The recycle delay time is $T R=1146 \mathrm{~ms}$. Right $\mathrm{B}$ ): combination of $\mathrm{A}$ ) with a spin-echo imaging sequence to obtain alanine selective planar images. $X$ and $Y$ dimensions are respectively encoded by frequency (X4 and X5) and (Y4) phase encoding gradients; P5 ( $\pi$ ) is a Gaussian shaped pulse, chemical shift selective on the alanine methyl resonance. $Z$ gradient is absent so that the image is taken throughout the entire volume. 
DQ alanine coherences by means of gradient pulses and transfers coherence from the methine to the observed methyl alanine resonance. In this way, the maximum in sensitivity for the alanine metabolite, together with a good water suppression, were reached. The sequence was then combined with a spin-echo imaging scheme (Scheme 1B), to give alanine selective imagcs. Spccific acquisition parameters are reported in the Scheme 1 caption, while experimental details are provided in figure captions.

Spin-echo imaging technique was preferred to gradient-echo, because fine structures inside small objects may often result in strong field inhomogeneities from susceptibility effects, which could degrade the achievable image resolution."

All 3D acquired data sets were processed using UXNMR, a Bruker software running on a X32 workstation and are presented in the paper not only as orthogonal planar sections of the insect, but also as photorealistic surface reconstruction.

All the experiments were carried out at room temperature. Silkworm larvae were fed on fresh mulberry leaves at $22-25^{\circ} \mathrm{C}$ in plastic containers. Whole silk glands were extracted from larvae and cleaned up from most of the adhering hemolymph before NMR analysis.

\section{RESULTS AND DISCUSSION}

Owing to the non-destructive nature of NMRI technique, it has been possible to follow and analyze the development of a single Bombyx mori during its complete life cycle, from young larva to moth, without introducing any intra subject variability into acquired data.

Figure 1 shows the photorealistic surface reconstruction of the insect in different life stages: larva (Fig. 1A), larva in the cocoon, studied few days after the spinning period (Fig. 1B), pupa (Fig. 1C) and moth (Fig. 1D). The cocoon does not give any image, being constituted by the silk thread winding made by fibroin and sericin, highly ordered as a consequence of the extrusion mechanical stress. Nevertheless, wetting the cocoon with water, it was possible to obtain an image from the protons of the absorbed water. Thus Fig. 1E shows the surface reconstruction of the whole envelope. The different insect stages will be separately considered.

\section{Larva}

Larva images have been acquired during one of the sleeping periods, after laying down with care the insect into a $20 \mathrm{~mm}$ NMR tube, where the larva assumed a partial folded position, well manifest in the image

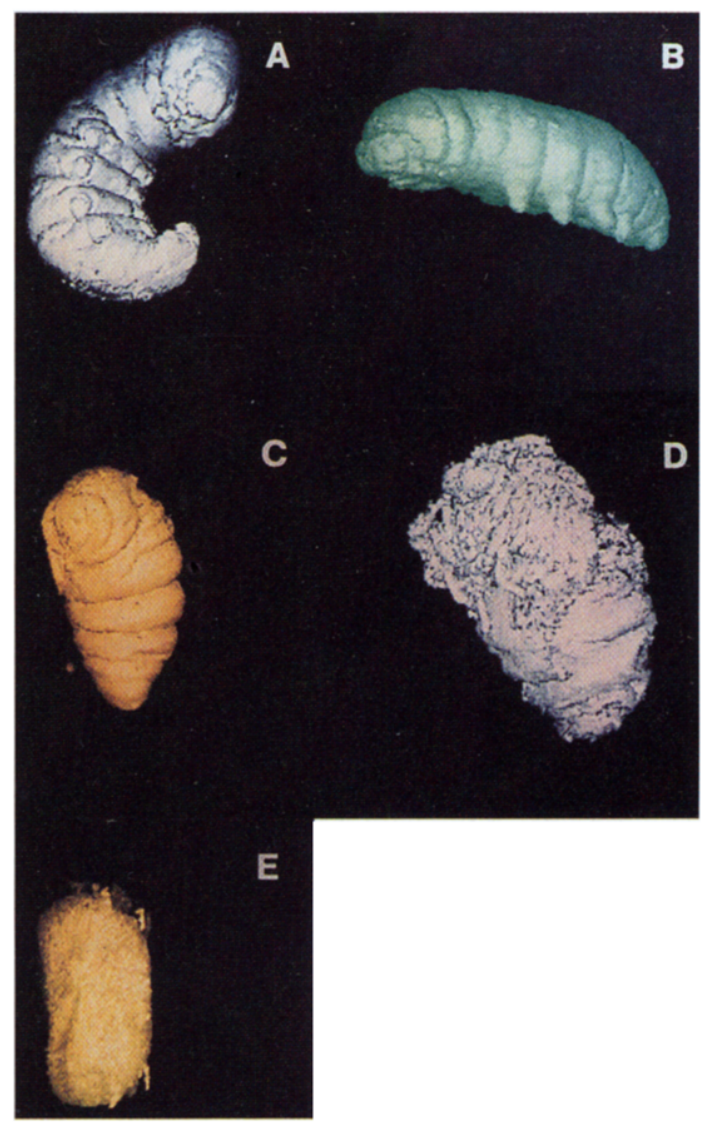

Fig. 1. Silkworm Bombyx mori 3D surface reconstruction of different life stages: A) young larva; B) larva in the cocoon; C) pupa; D) silk moth; E) 3D surface reconstruction of the cocoon obtained from the sample of B): the signal arises from spins of the water wetting the cocoon itself (see text). The photorealistic images come from processing of 3D spin-echo experiments.

reconstruction of Fig. 1A. As can be observed, the external morphology consists of metameric subdivisions, characteristic of insects, which allow three different regions of the body to be singled out: head, thorax and abdomen. In the head, the most noteworthy detail is the mouth masticatory apparatus, where it is possible to distinguish a rectangular membranous plaque forming the upper lip. In the thorax, each metamer is endowed with a pair of legs employed to approach leaves to mouth and appears prominent in the forepart, where numerous humps rich in skin pleats can be observed. Abdomen metamers, the last ones merged among them, are endowed with a pair of locomotory legs.

Figure 2 shows the internal morphology of the insect. The low contrast observed in the longitudinal and transverse slices ( $\mathrm{A}$ and $\mathrm{B}$ respectively), could derive from limited $T_{1}$ differences of the various tissues, or 

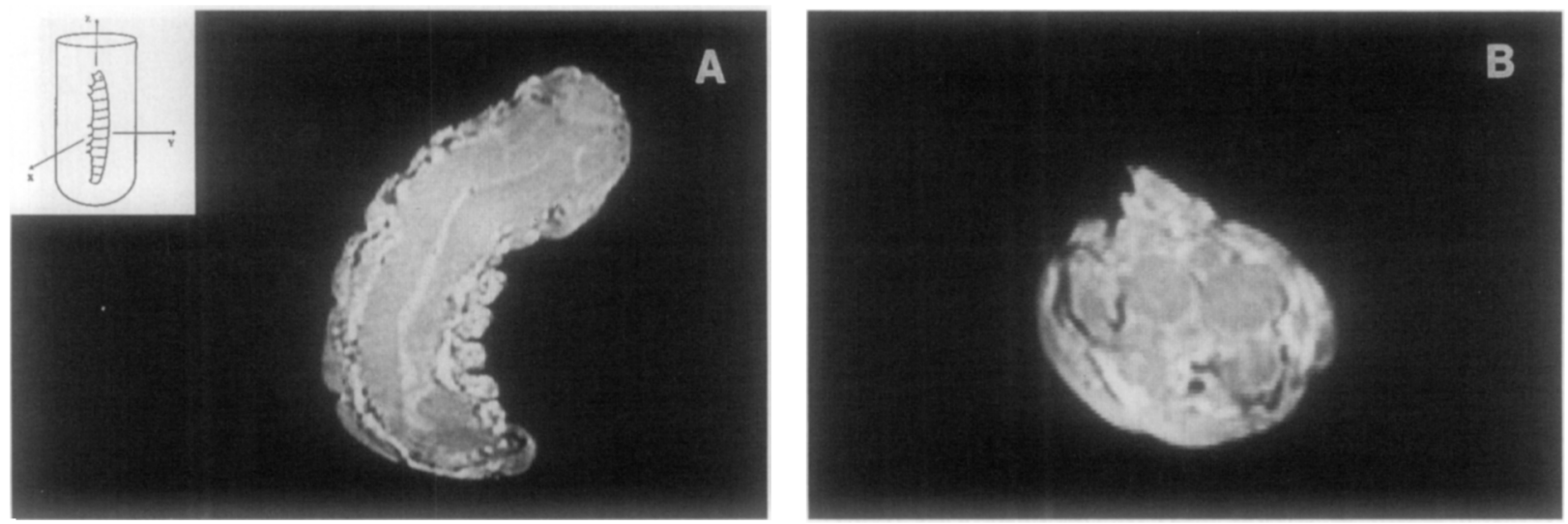

Fig. 2. Silkworm young larva: A) longitudinal and B) transversal slices from a 3D spin-echo experiment. $x y$-field of view (FOV): $21 \mathrm{~mm}$; z-field of view (LZ): $35 \mathrm{~mm}$; voxel resolution: $164 \times 164 \times 273 \mu \mathrm{m}^{3}$. For other acquisition parameters see text. The axis system relating to the orientation of the insect is also shown.

most likely from the TR value, chosen too short to give $T_{1}$ weighted images. Nevertheless the thin gut as well as the silk glands are well distinguishable in both the images. In particular, the silk glands can be singled out, owing to a slightly darker contrast. At this evolution stage, the water in the silk glands could be immobilized, as bound to the huge amount of fibroin synthetized by the posterior glands. This hypothesis would be in agreement with the mean correlation time (2.2 $\times 10^{-10} \mathrm{~s}$ ) of fibroin, measured by Asakura ${ }^{12}$ from partially-relaxed ${ }^{13} \mathrm{C}$ NMR spectra of a silk gland portion from intact larvae.

At the same evolution stage, the silk glands were extracted from a silkworm, cleaned up from most of the hemolymph and analyzed by high resolution NMR technique. Figure 3 shows the 1D proton spectra collected after the extraction. The spectrum of Fig. 3A, immediately collected, shows resonances from the most abundant fibroin amminoacids at 1.3 (Ala methyl), 3.9 (Gly $\mathrm{CH}_{2}$ ), 6.8 and 7.1 (Tyr aromatic protons), 8.2 and 8.4 (amide protons) ppm, in addition to the main water peak at $4.7 \mathrm{ppm}$. After few hours the water resonance appeared broader (Fig. 3B), even broader after about twenty-four hours (Fig. 3C), showing shoulders and a larger peak area which indicated that the gland content changed with time, after being taken out from glands. The interpretation of this fact is not trivial: it might be related to the fraction of water trapped in the fibroin that upon fibroin coagulation changed in mobility and then became detectable by NMR. ${ }^{14,15}$

With time, the water signal became so strong (Figure 4A) that the fibroin resonances could no more be distinguished by means of a single pulse sequence. Thus, ZQ and DQ filtered 1D NMR experiments were performed to select the resonance of alanine, one of the most abundant components of fibroin. As expected, the spectrum reported in Fig. 4B confirms the presence of alanine in silk glands at this stage. However, the slow segmental motion ${ }^{13}$ of the protein could likely be responsible for the impossibility of acquiring $Z Q$ and DQ filtered alanine selective images of the silk glands in vivo.

\section{Larva in the Cocoon}

From the surface reconstruction (Fig. 1B), it can be noticed that the external morphology of the silkworm larva inside its cocoon did not significantly change with respect to the previously analyzed stage.

On the contrary, as shown in Figure 5, both from longitudinal (Fig. 5A) and transverse (Fig. 5B) slices, the intcrnal morphology appears highly differentiated, owing to the striking contrast of the silk glands in comparison with the other organs. As previously mentioned, the chosen TR and TE did not optimize the image contrast, nevertheless, as the acquisition parameters were standardized for all 3D experiments, any difference in the contrast has to be related to changes in water properties. Indeed, in Fig. 5B a portion of the glands exhibits a higher intensity. This fact would be reasonably ascribed to different water mobility upon fibroin extrusion and to the onset of fibroinase activity. In fact, at this stage, the enzymatic activity is well known to be already started, ${ }^{8,9}$ so that the intermediates of fibroin, remained in the lumen content, possibly interacting with water, should have shorter correlation times. The fibroinase activity is most notably in the middle region of glands, which faster degenerated, showing the highest rate of decrease in wet weight after the spinning period. ${ }^{8}$

The intestines are not visible any more in the im- 
ages, probably due to their complete dumping that usually happens immediately before the cocoon formation.

\section{Pupa}

As shown in the surface reconstruction of Fig. 1C, silkworm pupa appears like a spindle, short and squat, devoid of appendices. Both the thick chitin layer wrapping the insect and the cocoon are not visible.

Compared to larva, pupa presents considerable differences in the three previously analyzed body regions, now less distinguishable. The fusion of some segments caused the number of metamers to be significantly decreased. Rudiments of the future legs, folded and stuck
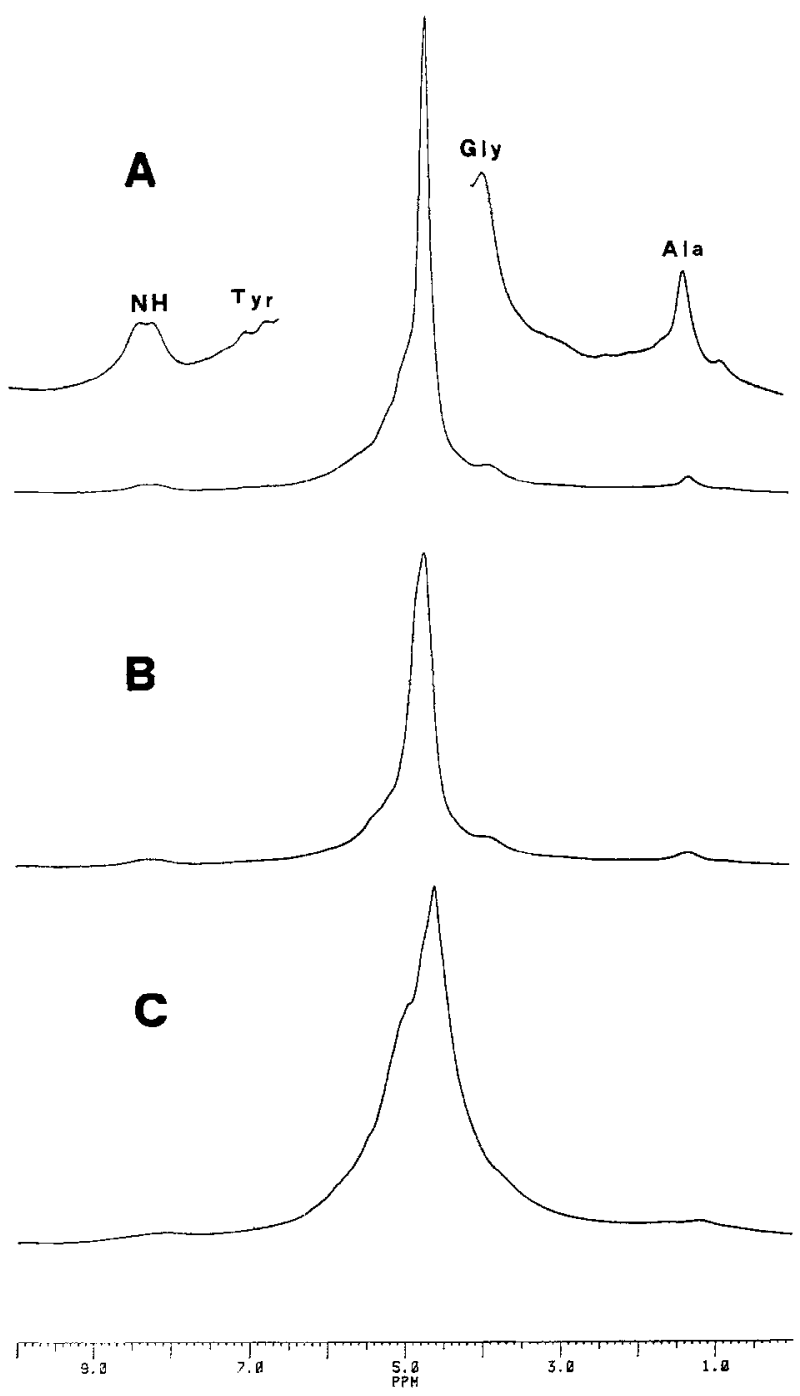

Fig. 3. Silk glands extracted from a silkworm. $1 \mathrm{D}{ }^{1} \mathrm{H}$ NMR spectra collected A) immediately after the extraction (the inserts are $\times 8$ vertical expansions), B) after three $h$ and $C$ ) after twenty-four h. Ala, Gly, Tyr and NH stand for alanine and glycine $\alpha$-protons, tyrosine aromatic protons and amide protons, respectively.

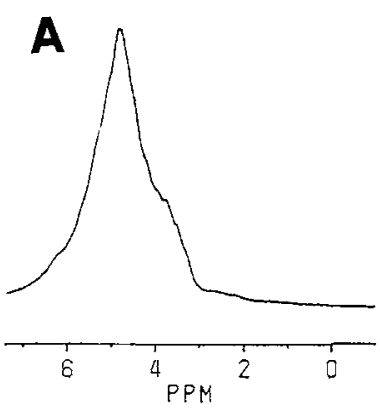

B

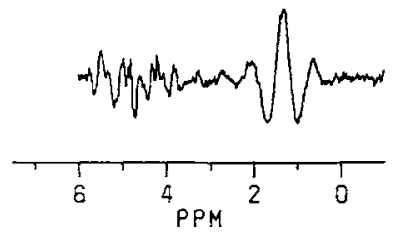

Fig. 4. Silk glands extracted from a silkworm. A) reference spectrum, recorded using a single pulse sequence $(\pi / 2=$ $12.7 \mu \mathrm{s}$ ); B ) $1 \mathrm{D} \mathrm{ZQ}$ and $\mathrm{DQ}$ filtered spectrum. $\mathrm{SW}=1845$ $\mathrm{Hz}$; NS $=256$. Standardized acquisition parameters are reported in Scheme 1A. A line broadening of $3 \mathrm{~Hz}$ was applied before Fourier transformation.

to the body, and of the wings are manifest. In the same way, the organization of internal organs changed, as it can be deduced from the longitudinal and transverse slices (Figures 6A and $\mathrm{B}$ ). The silk glands and intestines completely disappeared: in fact pupa functions
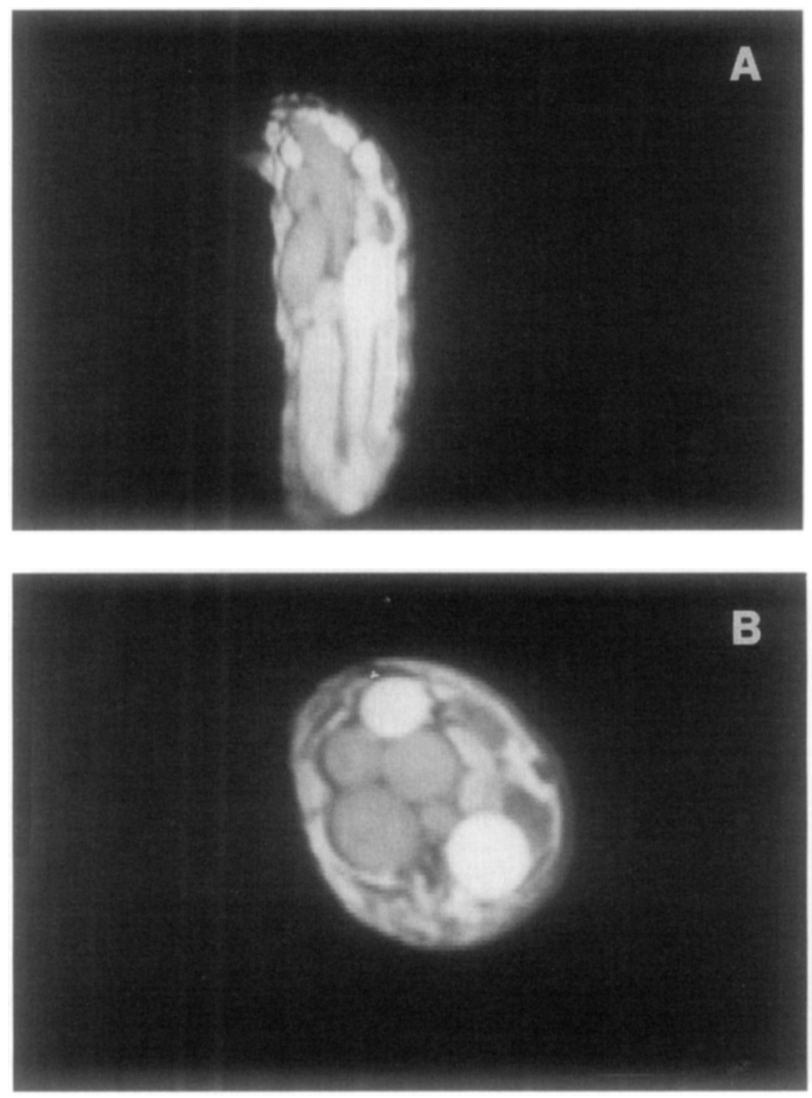

Fig. 5. Silkworm larva after the cocoon formation; A) longitudinal and B) transversal slices from a 3D spin-echo experiment. FOV: $21 \mathrm{~mm}$; LZ: $40 \mathrm{~mm}$; voxel resolution: $164 \times$ $164 \times 313 \mu \mathrm{m}^{3}$. For other acquisition parameters see text. 
are reduced only to a breathing activity and desultory muscle contractions. In Fig. 6A the separation between the head, darker, and the abdomen, characteristic of the adult insect, can be singled out. A few organs are probably rudiments of the complex genital apparatus that will reach the maximum development in the last stage.

\section{Adult}

Figure 1D shows the surface reconstruction of the adult silkworm or silk moth. The 3D data set has been acquired fifteen days later with respect to the pupa one, after having extracted the moth from its cocoon, as the insect could not spontaneously emerge. The silk moth does not look like a showy butterfly: the body is completely covered by a thick down that prevents the different body regions from being clearly identified. In the head the mouth apparatus disappeared, while the composite eyes, better observed in the transversal slice of Figure 7A, consist of two huge subspherical bawls. Three thoracic segments can be hardly recognized, endowed with a pair of thin locomotory legs, not present
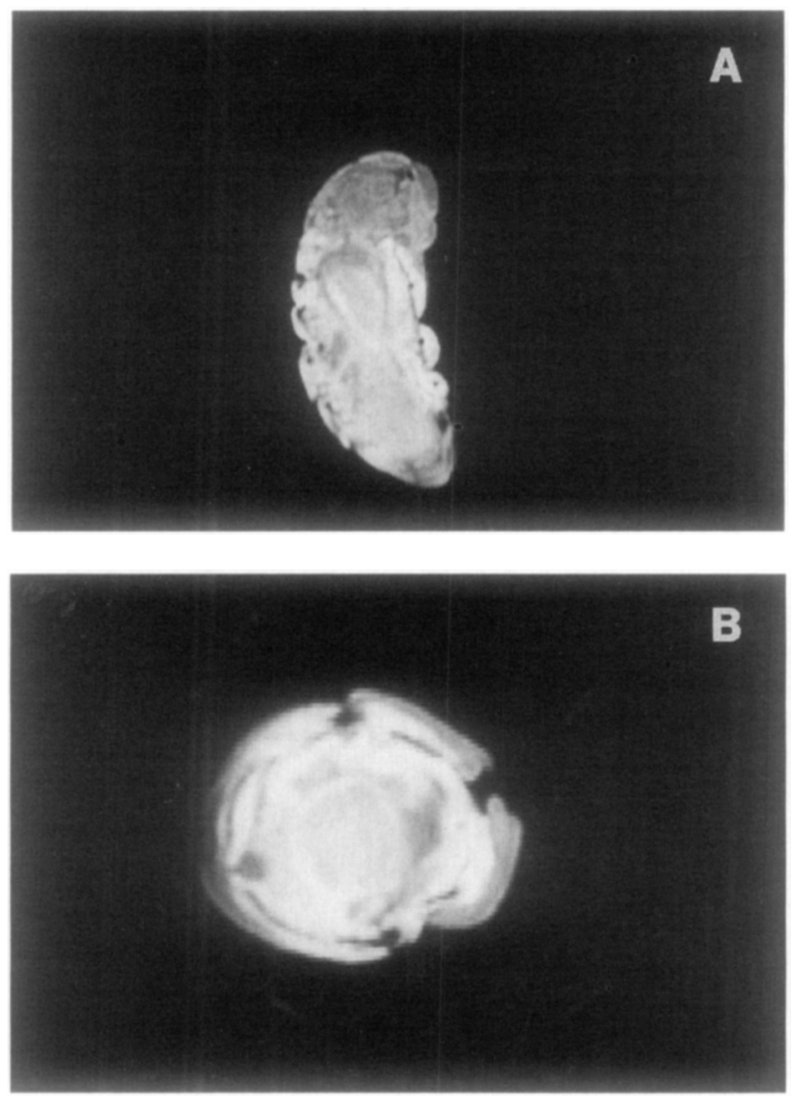

Fig. 6. Silkworm pupa: A) longitudinal and B) transversal slices from a 3D spin-echo experiment. FOV: $21 \mathrm{~mm}$; LZ: $40 \mathrm{~mm}$; voxel resolution: $164 \times 164 \times 313 \mu \mathrm{m}^{3}$. For the acquisition parameters see text.
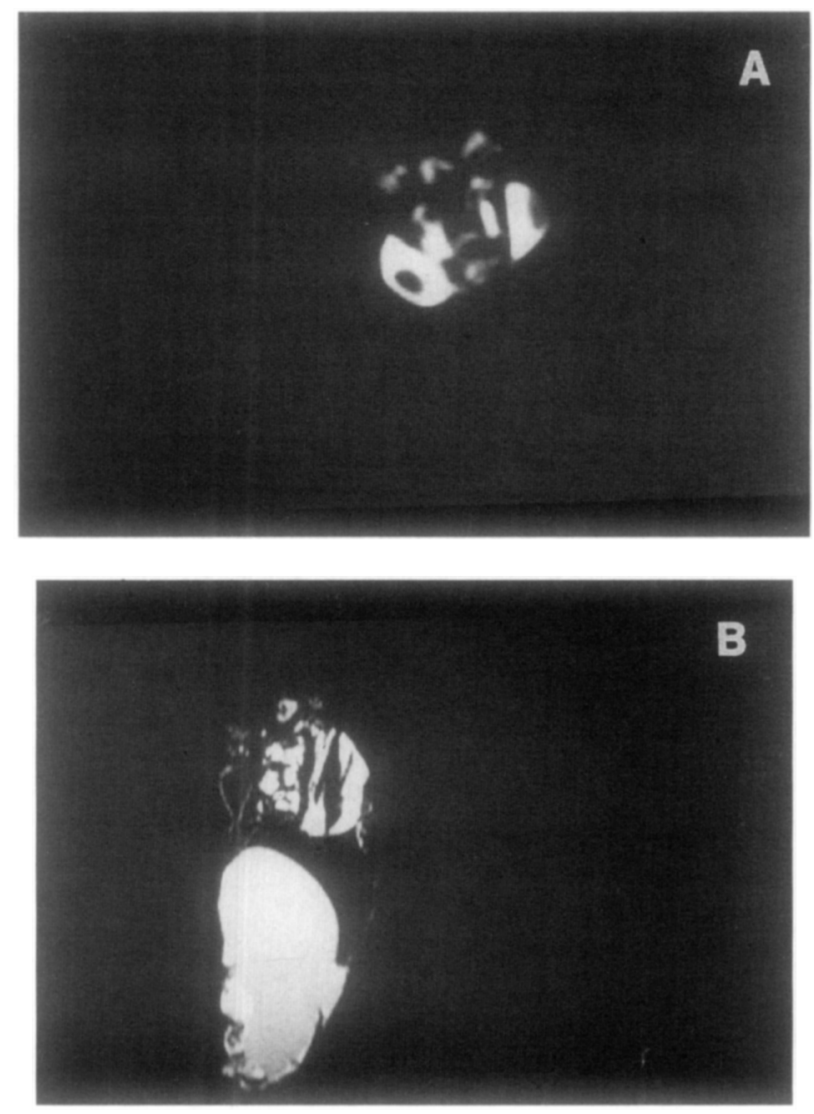

Fig. 7. Silkworm adult or silk moth: A) transversal slice of the composite eyes and $\mathrm{B}$ ) longitudinal slice of the moth from a 3D spin-echo experiment. FOV: $11 \mathrm{~mm}$; LZ: $30 \mathrm{~mm}$; voxel resolution: $86 \times 86 \times 243 \mu \mathrm{m}^{3}$. For other acquisition parameters see text.

in the abdomen any more. In the dorsal region, the wings are still folded on themselves, maintaining the same position they had in the cocoon and that as a matter of fact should have been changed outside the cocoon.

The internal morphology deeply modified, as can be observed in the longitudinal section reported in Figure $7 \mathrm{~B}$. The digestive system underwent radical transformations, the most notably being the presence of an air vesica, between the head and the abdomen, where an alkaline liquid was stored during the last days of the pupal stage and employed by the moth to wet and pierce the wall of the cocoon pole to emerge. In this case the cocoon was pierced and in fact, as clearly shown in Fig. 7B, the vesica is empty, suggesting that the moth tried to emerge without success. In the low region of the abdomen, the homogeneous and intense contrast is due to the large quantity of eggs that the moth should have laid. No connections are present between the head and the abdomen, except for very thin lateral ducts: the moth does not eat at all since its 


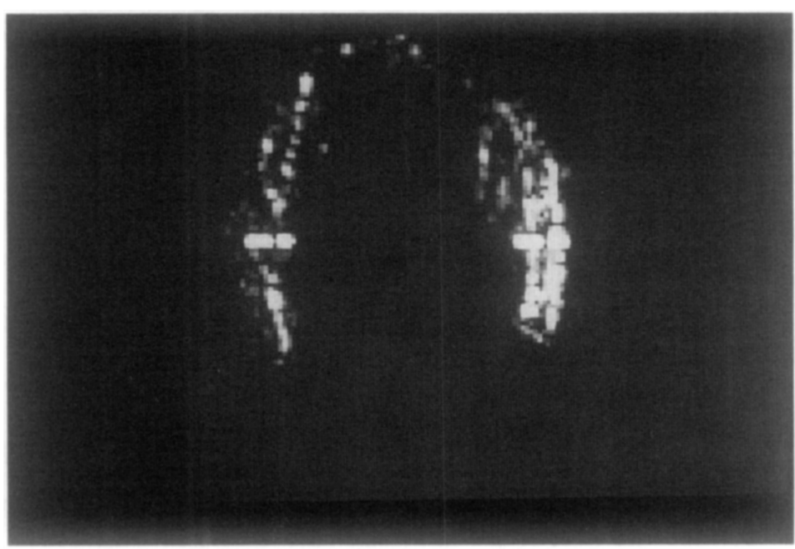

Fig. 8. ZQ and DQ filtered xy-image chemical shift selective on the methyl resonance of alanine obtained from the entire body of the silk moth. Pixel resolution: $86 \times 86 \mu \mathrm{m}^{2}$; NE $=400 ; \mathrm{SW}=9300 \mathrm{~Hz}$; echo delay time $(\mathrm{TE}): 165 \mathrm{~ms}$; recycle delay time $(T R): 1165 \mathrm{~ms}$.

feeding needs are satisfied by the material stored during the previous larva stage.

In order to check the presence of alanine among the possible fibroin intermediates and localize it inside the new body structure of the insect, an alanine ZQ and DQ filtered xy image of the whole moth body has been acquired (see Scheme 1B). The sequence, implemented on phantoms consisting of water, olive oil and lactate, ${ }^{10}$ was tested on a biphasic phantom consisting of water and alanine in deuterated water. Due to the sequence length $(\mathrm{TE}=165 \mathrm{~ms})$, the sensitivity of the experiment is quite low; anyway, the image reported in Figure 8 shows that alanine is still present in the last life stage of the insect. This image, projection of the whole silk moth body on the xy plane is orthogonal to the one reported in Fig. 7B. It indicales that the selected amino acid is localized in the external covering of the body, where most likely the fibroin metabolites have been employed to form new tissues. On the contrary, alanine is absent in the central region of the body occupied by the insect eggs.

\section{CONCLUSIONS}

In the present study NMRI was used to obtain, in a non-invasive way, morphological information on the internal structure of silkworm, Bombyx mori, during its postembryonal metamorphosis through the larva, pupa and adult stages. Image analysis allowed to visualize, on a single silkworm sample, the gradual modification of the internal organs, particularly focusing on intestines and silk glands development and on the parallel metamorphosis of the external covering. Be- sides, it provided a detailed picture of the distribution of the alanine residue, a metabolite of fibroin and sericin, the major fibrous constituents of the silk glands. NMRI allowed to monitor differences in water properties in the silk glands, related to changes in the mobility of water, upon the extrusion of fibroin and the onset of the fibroinase activity, especially in the middle region of the glands. Finally, a ZQ and DQ image showed that, in the last life stage of the insect, alanine is localized in the external covering of the moth body, where most likely the fibroin metabolites are utilized. These results suggest that the NMRI approach could be very helpful in studies aimed at investigating the metabolism of chimeric fibroin in genetically modified silkworm species, as the ones proposed by Mori et al. ${ }^{16}$

Acknowledgments - The authors would like to express their grateful thanks to Dr. A. Seves of Stazione Sperimentale Cellulosa, Carta e Fibre Tessili of Milano for helpful discussion, Dr. S. Faragò of Stazione Sperimentale della Seta of Milano for helpful discussion and for providing silkworms, Dr. N. Masciocchi of the University of Milano for taking care of the photographic work, Mr. G. Zannoni of ICM, CNR Milano for technical assistance and the "Associazione Antonio De Marco" for financial support.

\section{REFERENCES}

1. Callaghan, P.T. In: Principles of NMR Microscopy. Oxford, UK: Clarendon Press; 1991: pp. 228-337.

2. Blümich, B.; Kuhn, W. In: Magnetic Resonance Microscopy. Methods and Application in Materials Science, Agriculture and Biomedicine. VCH; 1992: pp. 165573.

3. Gassner, G.; Lohman, J.A.B. Combined proton NMR imaging and spectral analysis of locust embryonic development. Proc. Natl. Acad. Sci. USA 84:5297-5300; 1987.

4. Listerud, J.M.; Sinton, S.W.; Drobny, G.P. NMR imaging of materials. Anal. Chem. 61:23-41; 1989.

5. Goodman, B.A.; Gordon, S.C.; Chudek, J.A.; Hunter, G.; Woodford, J.A.T. Nuclear-magnetic-resonance microscopy as a non-invasive tool to study the development of Lepidopteran Pupae. J. Insect Physiol. 41:419424; 1995.

6. Skibbe, U.; Christeller, J.T.; Eccles, C.D.; Laing, W.A.; Callaghan, P.T. Phosphorus imaging as a tool for studying the $\mathrm{pH}$ metabolism in living insects. J. Magn. Reson. B 108:262-268; 1995.

7. Sehnal, F.; Akai, H. Insect silk glands: Their types, development and function, and effects of environmental factors and morphogenetic hormones on them. Int. J. Insect Morphol. Embryol. 19:79-132; 1990.

8. Sumida, M.; Takimoto, S.; Matsubara, F. Fibroinase from silk gland in the fourth molt stage in the silkworm Bombyx mori. Comp. Biochem. Physiol. 105B:247$251 ; 1993$.

9. Sumida, M.; Takimoto, S.; Ukai, M.; Matsubara, F. Occurrence of fibroinase in degenerating silk gland in the 
pharate adult of the silkworm Bombyx mori. Comp. Biochem. Physiol. 105B:239-245; 1993.

10. Gussoni, M.; Vezzoli, A.; Greco, F.; Consonni, R.; Pegna, M.; Molinari, H.; Zetta, L. NMRI studies on phantoms for an unequivocal determination of lactate in tissues by ' $\mathrm{H}$ spectroscopy. Quart. Magn. Res. in Biol. and Med. 1:39-45; 1994.

11. Ahn, C.B; Chu, W.C. Optimal imaging strategy for three-dimensional nuclear magnetic resonance microscopy. J. Magn. Reson. 94:455-470; 1991.

12. Asakura, T. NMR of silk fibroin, structure of Bombyx mori silk fibroin in aqueous solution. Makromol. Chem., Rapid Commun. 7:755-759; 1986.

13. Hore, P.J. Solvent suppression in Fourier transform nu- clear magnetic resonance. J. Magn. Reson. 55:283-300; 1983.

14. Magoshi, J.; Magoshi, Y.; Nakamura, S. Crystallization, liquid crystal and fiber formation of silk fibroin. J. Appl. Polym. Sci. 41:187-204; 1985.

15. Akasura, T.; Demura, M.; Watanabe, Y.; Sato, K. 'H pulsed NMR study of Bombyx mori silk fibroin: dynamics of fibroin and of absorbed water. J. Polym. Sci. Polym. Phys. 30:693-699; 1992.

16. Mori, K.; Tanaka, K.; Kikuchi, Y.; Waga, M.; Waga, S.; Mizuno, S. Production of a chimeric fibroin lightchain polypeptide in a fibroin secretion-deficient naked pupa mutant of the silkworm Bombyx mori. J. Mol. Biol. 251:217-228; 1995. 